Canadian University Music Review

Canadian University Music Review

Revue de musique des universités canadiennes

\title{
Différence et similitude dans les Préludes pour piano de Debussy
}

\section{Marcelle Guertin}

Numéro 2, 1981

URI : https://id.erudit.org/iderudit/1013744ar

DOI : https://doi.org/10.7202/1013744ar

Aller au sommaire du numéro

Éditeur(s)

Canadian University Music Society / Société de musique des universités canadiennes

ISSN

0710-0353 (imprimé)

2291-2436 (numérique)

Découvrir la revue

Citer cet article

Guertin, M. (1981). Différence et similitude dans les Préludes pour piano de Debussy. Canadian University Music Review / Revue de musique des universités canadiennes, (2), 56-83. https://doi.org/10.7202/1013744ar

All Rights Reserved @ Canadian University Music Society / Société de musique des universités canadiennes, 1981
Ce document est protégé par la loi sur le droit d'auteur. L'utilisation des services d’Érudit (y compris la reproduction) est assujettie à sa politique d'utilisation que vous pouvez consulter en ligne.

https://apropos.erudit.org/fr/usagers/politique-dutilisation/ 


\title{
DIFFÉRENCE ET SIMILITUDE DANS LES PRÉLUDES POUR PIANO DE DEBUSSY
}

\author{
Marcelle Guertin
}

\section{L'énigme originelle}

Lorsque, il y a de cela une décennie environ, je m'installai au piano pour ouvrir et découvrir le Livre I des Préludes de Debussy, j'ignorais qu'à plusieurs années de là, je serais amenée à faire, sur ce même recueil, d'autres genres de découvertes, analytiques cette fois, mais dont les fondements tireraient leur origine de certaines impressions éprouvées en ce lointain aprèsmidi. Impression du stable et de l'instable, du persistant et de l'évanescent dans ces pages qui se ressemblent sans se ressembler. Impression d'équivoque, de paradoxe; d'un toujours pareil et d'un toujours différent dans ces jeux sonores ondoyants et dans les gestes des mains sur le clavier. Je faisais alors l'apprentissage empirique d'un corpus dont je serais appelée à définir certains aspects par la suite, dans le cadre d'une recherche en sémiologie musicale. Mais déjà à ce moment-là, et bien que flottant voluptueusement dans les voiles du non-dit entre Delphes et Anacapri, j'avais l'intuition d'un même faire, toujours recommencé et toujours remis à neuf, traversant de part en part les pièces homogènes-hétérogènes de ce recueil; l'intuition, c'est-à-dire, selon le mot de Bergson, " une sorte de sympathie " avec des évidences qui ne me furent pourtant révélées comme telles que bien plus tard, après une étude approfondie de la mélodie dans les Préludes du Livre I.

Bien sûr, au fil des années et de la fréquentation de la musique de Debussy, j'ai appris à connaître plus rationnellement certains procédés chers à ce compositeur, tant au niveau des échelles utilisées, des brouillages harmoniques obtenus au moyen d'accords altérés, de suspensions, de pédales, de décentrations tonales subites, qu'à celui d'un traitement spécifique 
des timbres et des registres apte à donner cette coloration sonore par laquelle on reconnaît immédiatement Debussy. Mais cela ne suffisait pas à décanter mes intuitions originelles. Quand je rejouais les Préludes du Livre I ou que je les écoutais sur disques, je ne pouvais m'empêcher de me demander d'où il vient que, d'un morceau à l'autre, cette musique se dérobe en même temps qu'elle se fixe; par quel subterfuge elle se transmue constamment tout en demeurant identique à elle-même. A ces questions, je répondais tant bien que mal, me disant qu'il ne s'agissait pas là d'un privilège des Préludes, mais que c'est le propre de toute musique de se dérober - puisqu'elle se déroule dans le temps suivant le destin inéluctable des minutes et des secondes écoulées - tout en se fixant et en se construisant en nous sous l'emprise de la mémoire. Et c'est aussi le propre de nombre de pièces réunies dans un recueil que de présenter. à la fois des caractères communs et distinctifs, surtout lorsque, comme ces Préludes, elles ont été composées à la même période ou ont, à leur source, un même souci poïétique. Or, ces réponses n'étaient pas pour me satisfaire, car elles ne faisaient que déplacer et généraliser le problème, posé, en ce cas-ci, sur un ensemble déterminé de pièces.

Que cherchais-je donc? J'avais le sentiment que cette dialectique du "fugitif " et du "durable ", du "pareil » et du "différent ", si fortement ressentie à l'audition des Préludes, n'était pas qu'un des effets éthérés des sortilèges de cette musique, non plus que le seul produit de la récurrence, ici et là, de procédés aisément repérables : gammes par ton, échelles modales, doublures à l'octave creusées par des quintes à vide ou troublées par des frottements de secondes, emploi synchrone des registres extrêmes, etc.; j'avais le sentiment que cette dialectique devait reposer sur des subterfuges justement, sur des particularités d'écriture qui, invariantes dans certains de leurs traits, dissimulaient habilement leur identité sous des traits changeants. A travers ces douze Préludes semblaient se réfugier des squelettes d'organisation suffisamment bien articulés pour que, d'une pièce à l'autre, et en dépit des particularités de chacune, subsistât cette impression si forte d'air de famille.

On le voit: c'est la fameuse question du style qui me préoccupait. Du style abordé sous un angle bien spécifique : celui du jeu simultané de la permanence et du changement à travers un corpus. Du style en tant qu'ensemble de processus 
fixes, structures sous-jacentes immuables appelées à se réaliser selon des modalités diverses. Du style en tant que dichotomie entre face visible multiple et face cachée uniforme. Certes, la formulation de cette question était alors loin d'être aussi claire qu'elle peut l'être maintenant, à bien des années de distance, à la lumière d'une recherche systématique ayant abouti à des résultats concrets et à une mise en forme sur le plan de l'écriture. Et ce serait un leurre de prétendre qu'elle fût vraiment posée à ce moment-là; tout au plus s'agitait-elle dans les ténèbres de sensations et de pressentiments ruminés dont je puis dire maintenant, et maintenant seulement, au terme d'une analyse passée au sas du langage, qu'ils ressortissaient cependant de ce type d'interrogation.

C'est lors de la création, en 1974, du Groupe de recherches en sémiologie musicale de l'Université de Montréal, que j'ai été amenée à travailler sur les Préludes pour piano. L'objectif du projet consistait à tester des méthodes similaires d'analyse sur deux corpus différents : l'un de tradition orale - les jeux de gorge ou « katajjait » des Inuit du Nouveau-Québec - , l'autre appartenant au répertoire occidental - le premier Livres des Préludes de Debussy. Il n'entre pas dans mon propos de faire état du travail effectué par mes collègues sur le "katajjaq "; mais il n'est peut-être pas inutile de rappeler que, au-delà des tâches propres aux ethnomusicologues, l'esprit de la démarche a été le même pour l'analyse des deux corpus, démarche dont je vais présenter les principales constituantes au cours de cet article.

\section{Une certaine approche stylistique}

Dans une lettre adressée à André Messager un an et demi après la fin de son opéra, Debussy confiait :

Quant aux personnes qui me font l'amitié d'espérer que je ne pourrai jamais sortir de Pelléas, elles se bouchent l'œil avec soin. Elles ne savent donc point que, si cela devait arriver, je me mettrais immédiatement à cultiver l'ananas en chambre, considérant que la chose la plus fâcheuse est bien de "se recommencer" (lettre du 12 septembre 1903 in Messager 1938 : 74).

"Se recommencer": voilà une phobie bien terrible en vérité pour quiconque est tant soit peu obsédé par les pièges de la répétition en abordant une composition nouvelle. Mais 
d'autre part, et sans que celle-ci soit un calque de la précédente - car il y a répétition et répétition -, comment éviter le phénomène d'un recommencement quelque part, phénomène inexorable s'il en est un, et auquel personne ne semble avoir échappé jusqu'ici? Impossible bien sûr, et Debussy le savait fort bien, qu'on voit mal abandonner la composition musicale pour les délices d'une culture fruitière, fût-elle parfumée d'exotisme.

Dans la production de tout individu, les constantes sont inévitables, qu'elles se manifestent ouvertement ou clandestinement. C'est, entre autres, leur identification qui permet l'établissement d'une stylistique. C'est aussi, dans une large part, sur leur existence que s'appuient, à l'écoute d'une pièce dont on ignore le signataire, des déclarations du genre : "Tiens, du Debussy ». De quoi est-elle signe, cette métonymie, sinon de la re-connaissance, implicite ou explicite, d'une "manière " particulière, et particulièrement forte, cristallisée dans des gestes réitérés? Et qu'arrive-t-il lorsque, au hasard d'une pièce du même compositeur, ces gestes ne se retrouvent pas? Eh bien, l'étiquette ne colle plus, et l'on s'écrie tout bêtement : "Mais ça n'est pas croyable! C'est Debussy qui a fait ça?"

De la façon la plus globale qui soit, le concept de style renvoie à un mode déterminé de traitement de la matière. Mais une telle définition, par son degré de généralité et d'abstraction, demeure inintéressante hors contexte. Elle demande à être raffinée à l'intérieur d'une démarche analytique précise qui, compte tenu du corpus étudié et des variables considérées, sera susceptible de lui faire prendre des visages fort différents. C'est ainsi que, fonction d'une approche spécifique, le style pourra être défini tour à tour comme norme, comme déviation par rapport à une norme, comme choix opéré par toute pièce parmi un nombre $\mathrm{x}$ de possibilités, ou encore par opposition à un autre style.

Il me m'appartient pas d'entrer dans le détail de ces diverses acceptions - la tâche est au-dessus de mes compétences - non plus que de juger de leur valeur respective. Je m'attacherai cependant à préciser la position qui est la mienne et qui a dicté la voie dans laquelle j'ai engagé l'enquête sur les Préludes de Debussy: le style en tant que norme régissant, par-delà les caractéristiques ponctuelles et distinctives, certains aspects de la facture d'un corpus.

Pour un groupe d'ouvres choisies, l'enjeu consiste à définir cet "air de famille » dont je parlais précédemment, et ce, à 
l'aide d'une procédure aussi bien comparative que quantitative, axée sur la notion de réitération : parmi la foule des traits qui composent chaque pièce du champ d'investigation, il s'agit de laisser de côté les traits singuliers - ceux qui font le propre de chaque morceau ou qui n'apparaissent que sporadiquement à travers le corpus - pour ne retenir que ceux qui sont communs à l'ensemble ou, du moins, à la majorité. Entendons-nous sur le statut de ces traits communs : la communauté de traits ne signifie pas identité entre les éléments retenus, bien que cela puisse arriver à l'occasion; elle signifie plutôt que, considérés sous l'angle d'une variable (rythme, hauteurs, harmonie, forme, etc.) ou d'une combinaison de variables, ces éléments reposent, en dépit de leurs particularités, sur un même mécanisme de base, sur un principe général d'organisation. ${ }^{1}$ Une fois dégagés, les traits communs sont convertis en système de règles, dites "normatives» parce qu'elles rendent compte d'un aspect courant, constant dans le mode de traitement du matériau.

Une norme sera plus ou moins rigide selon l'ampleur du corpus étudié, selon que l'on s'attaque à la musique française du début de ce siècle, à l'œuvre de Debussy, ou au seul Livre I de ses Préludes pour piano. Et, à l'intérieur de ce corpus, elle dépendra aussi de tous les processus de sélection opérés par l'analyste: sur quel(s) phénomène(s) particulier(s) il s'est penché, par le biais de quelle(s) variable(s), à l'appui de quels critères, et en regard de combien de cas précis. Face à quelque loi stylistique proposée, ces considérations ne devraient jamais être perdues de vue, tant il est vrai que l'établissement de toute norme relève d'une démarche analytique partielle et partiale : celle qui, taillant constamment dans le corpus avec un petit couteau bien personnel qui s'appelle "point de vue », privilégie des portions au détriment d'autres.

Mais, pour un corpus donné, existe-t-il des points de vue plus pertinents que d'autres? Incapables de répondre à cela, je serais cependant tentée de dire : non. Les chemins analytiques sont ouverts, comme ouvertes les ouvres leur servant de support. Dans l'infinité des optiques possibles, il y en a peutêtre qui sont plus convaincantes que d'autres, mais, aussi divergentes qu'elles soient, toutes ces approches n'en demeurent pas moins complémentaires, projetant, chacune à sa façon, un mode de préhension spécifique des objets qu'elles examinent. Dans son avant-propos à Sur Racine, Roland Barthes formule la problématique en termes incitant à la réflexion - il traite du 
champ littéraire, mais il nous est facile d'opérer le transfert dans le domaine musical :

Écrire, c'est ébranler le sens du monde, y disposer une interrogation indirecte, à laquelle l'écrivain, par un dernier suspens, s'abstient de répondre. La réponse, c'est chacun de nous qui la donne, y apportant son histoire, son langage, sa liberté; mais, comme histoire, langage et liberté changent infiniment, la réponse du monde à l'écrivain est infinie : on ne cesse jamais de répondre à ce qui a été écrit hors de toute réponse : affirmés, puis mis en rivalité, puis remplacés, les sens passent, la question demeure (Barthes 1963 : 11).

A toutes les interrogations indirectes suscitées par la musique de Debussy, j'ai choisi, quant à moi, d'apporter des bribes de réponse au niveau des structures mélodiques, et l'objet de mon investigation est le suivant : UNE STYLISTIQUE DE LA FORME DANS LES THËMES DU PREMIER LIVRE DES PRÉLUDES POUR PIANO.

\section{Les 'a priori' de l'enquête}

Dès l'abord, mon objet de recherche comporte un terme ambigu: le mot "thème ". Des nombreuses significations fournies par la terminologie officielle, la plus opératoire dont j'ai cru pouvoir me servir est celle-ci ${ }^{2}$ : est appelé " thème " un énoncé à la fois mélodique et rythmique, perçu comme entité, et destiné à être développé au cours de la composition dans laquelle il figure; mais il n'y a pas que des thèmes mélodicorythmiques : certains peuvent être axés sur le rythme seul, d'autres encore, sur un ou plusieurs complexes harmoniques.

De cette définition, j'ai décidé de ne garder que quelques éléments pour mon analyse. Désirant porter mon attention sur la dimension horizontale de l'écriture, j'ai écarté d'emblée les phénomènes verticaux. Je n'ai conservé que le principe d'événements linéaires à la fois mélodiques et rythmiques, considérés comme tout homogène, comme Gestalt, excluant toutefois les lignes d'ostinato et d'accompagnement. Est donc retenu dans mon champ analytique tout objet à forte prégnance mélodique, auquel la perception - la mienne en l'occurence - accorde le statut de thème.

Il va sans dire que cette définition n'en est pas vraiment une; scientifiquement, elle est inacceptable, puisqu'elle relève d'une appréciation subjective de la qualité d'un phénomène 
plutôt que de sa mesure objective. Cependant, comme la notion de " thème " s'inscrit, à mon avis, parmi les catégories de notre vécu musical occidental, elle mérite d'être retenue. Pour l'instant, il s'agit encore d'un quasi-concept, c'est-à-dire d'un concept semi-intuitif, aux contours flous, qui n'a pas été marqué du sceau de la réduction scientifique. ${ }^{3}$ Mais on peut espérer qu'une approche formalisée, effectuée sur un corpus délimité et aboutissant à un ensemble de règles, permette l'émergence d'un peu de clarté dans tout ce flou.

Continuant de faire le procès de mon quasi-concept, j'ai rejeté dès le départ l'affirmation selon laquelle un énoncé dit " thématique " doit être développé, c'est-à-dire, selon la conception traditionnelle, réapparaître dans le courant du morceau sous des formulations plus ou moins variées. Premièrement, parce que ce trait est superflu pour définir le mot " thème ": il n'aide en rien à en préciser le sens immanent, puisque tout élément autre qu'un thème est passible de développement. Deuxièmement, parce que, dans les pièces de mon corpus, certains thèmes ne réapparaissent pas au-delà de leur exposition. Enfin, parce que la notion de " développement " est très délicate à manier chez Debussy. Je n'irais pas jusqu'à dire qu'elle est impropre, mais elle exige une révision de ses prémisses et une distinction de cas de figures.

Il faut bien se rendre compte en effet qu'une grande partie du vocabulaire légué par la tradition musicologique est inapte, tout particulièrement dès que l'on franchit le seuil du XXe siècle, à nommer convenablement les phénomènes qu'il prétend englober. N'oublions pas que ce vocabulaire, né de l'observation d'une tranche limitée de la production musicale, est entaché chronologiquement; il est historique. Et déjà, dans son historicité, il pêche bien souvent par ambiguïté. Je pense ici, non seulement au mot "développement " - utilisé d'abord dans le cadre de la forme-sonate, puis appelé ensuite à transgresser ce cadre pour désigner maints phénomènes hétéroclites -; je pense également non seulement au mot "thème ", mais à tous ces motifs, cellules, membres de phrases, phrases, périodes, etc., lesquels, confrontés sur le plan de leur sens respectif et de leur emploi dans les œuvres en arrivent à ne plus pouvoir répondre de rien. Qui a dû se débattre avec pareil lexique, à l'intérieur d'une démarche pédagogique par exemple, sait fort bien de quoi il retourne. Ce vocabulaire exige une sérieuse 
remise en question; il appelle une attitude fondamentalement critique.

Après toutes ces invectives, il convient que je m'explique sur l'emploi têtu du mot "thème " dans mon travail. Disons d'abord que ce n'est pas tant au mot lui-même que je tiens - un autre mot le remplacerait tout aussi bien - mais au phénomène perceptif auquel il fait référence chez moi comme chez quantité de personnes. Par ailleurs, à l'intérieur d'une démarche explicitée, l'emploi du terme n'a pas à nous troubler outre mesure si, d'une part, les événements qu'il désigne sont clairement délimités, et si, d'autre part, ces événements font l'objet d'une description. Ce qui est contestable dans la terminologie traditionnelle, c'est son statut de Bible, et sa propension à favoriser le " nominalisme » : ceci est un motif ceci est une phrase - ceci est une chaîne de phrases - et ainsi de suite. Je suis bien prête à reconnaître à ces termes une valeur de quasi-concept, à condition toutefois que le travail analytique ne s'arrête pas une fois qu'on a tout bonnement nommé les choses. Face à ce danger de "nominalisme ", comment la sémiologie musicale a-t-elle procédé? Je serais tentée de répondre laconiquement : par la rigueur.

Sans prétendre dresser ici le bilan de tout ce qu'une approche sémiologique implique au niveau de l'analyse de partitions, je citerai toutefois quelques-uns de ses apports essentiels, intégrés dans ma propre démarche. Dans l'ordre, j'aborderai les trois points suivants, lesquels définissent par ailleurs des étapes successives de mon travail : la technique paradigmatique; l'explicitation des procédures de découverte; la systématisation du concept de méta-langage.

\section{a. La technique paradigmatique}

Celle-ci consiste à la fois en une méthode de découpage du continuum musical en unités, et en un mode d'agencement de ces unités à l'intérieur d'axes d'équivalence appelés paradigmes. Afin de mieux comprendre de quoi il retourne, voyons une application concrète de cette technique, en prenant comme exemple le thème qui ouvre le premier des Préludes du Livre I, Danseuses de Delphes. Des mesures 1 à 4 , ce thème apparaît comme suit : 
64

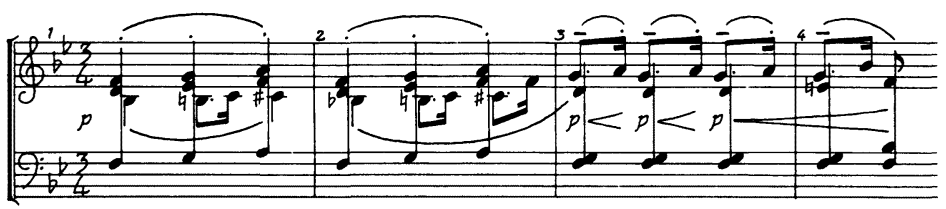

Exemple 1

Pour fins de commodité, il a été réduit à ses lignes principales, élagué de ses doublures :

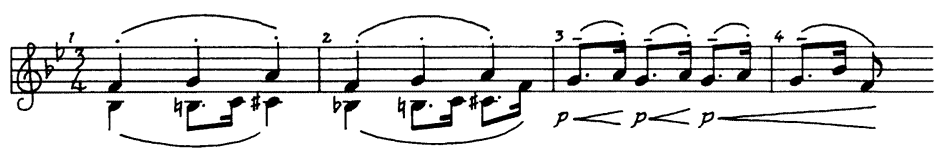

Exemple 2

Traité selon une application possible de la technique paradigmatique, il se présente ainsi :

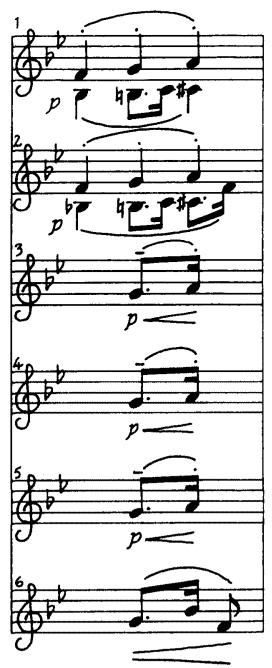

Exemple 3

Il se trouve segmenté en six unités, qui, parcourues de gauche à droite et de haut en bas, permettent de le reconstituer intégralement. La segmentation repose sur un critère d'identité ou de similarité mélodico-rythmique. La première unité est délimitée 
de la manière que l'on voit à cause de sa répétition par une seconde unité, laquelle, tout en la transformant par un ajout à la voix inférieure, n'en reprend pas moins le trajet mélodicorythmique. Une troisième unité suit, associée à la finale des deux premières parce qu'elle répète les sons sol-la de la voix supérieure - modifiés rythmiquement par les valeurs croche pointée-double, jusque-là propriété de la voix inférieure. Une quatrième, puis une cinquième unités sont copies conformes de la troisième. Enfin, une sixième unité se superpose aux précédentes à cause de sa rythmique d'abord, puis de son mouvement mélodique considéré comme renversement de celui des unités 3, 4 et $5:$ au lieu de sol-la, on a ici sol-fa séparés par une échappée (si bémol). Ces six unités sont, de l'une à l'autre, intimement liées, et leur superposition, fondée sur une conjugaison des variables " hauteurs ", "intervalles " et " durées", a pour but de montrer cette complicité. Réunies sur un même axe d'équivalence, elles forment donc paradigme, et ce, à partir d'un événement initial (l'unité 1) qui s'appelle tête de paradigme.

Le découpage effectué ici n'est évidemment pas le seul que l'on puisse pratiquer sur ce thème - et il en va de même pour tous les découpages qui seront présentés ultérieurement. Une segmentation comme celle-ci par exemple :

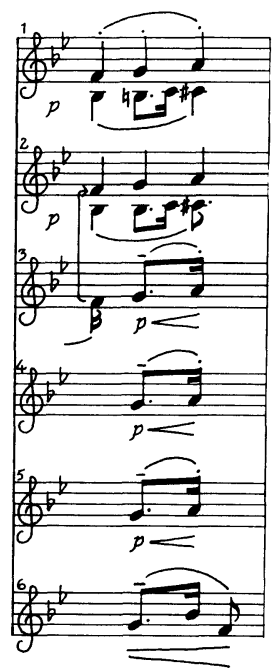


est tout aussi valable; par la délimitation différente de la troisième unité, elle fait intervenir un autre point de vue sur l'objet. Car, si paradigmatiser veut dire associer, la démarche sous-entend, parmi les multiples angles d'association possibles, des choix de la part de l'analyste. Mes choix sont essentiellement dictés par deux choses : par la perception que j'ai de chacun des thèmes étudiés, et, au-delà de chacun, par la décision de pratiquer sur l'ensemble un découpage homogène, apte à faire ressortir des traits communs.

La technique paradigmatique consiste donc à dresser une liste d'unités jugées équivalentes. Cependant, elle ne constitue qu'un premier pas dans le travail. Pour que les tableaux qu'elle suscite soient vraiment parlants, il faut, justement, que l'analyste parle, c'est-à-dire qu'il les explique. Et j'en arrive à mon deuxième point :

\section{b. L'explicitation des procédures de découverte.}

Je dirais qu'il s'agit là d'une condition sine qua non à toute entreprise analytique qui se veut sérieuse : c'est, en effet, dans la mesure où l'on explicite le pourquoi et le comment des rapports posés entre signes et ensembles de signes qu'un travail atteindra un certain statut scientifique. Les critères de sélection, de découpage, de mise en relation doivent être clairement formulés; d'abord, pour répondre à une exigence de systématicité et d'homogénéité dans la recherche; ensuite, pour permettre une validation des résultats obtenus; et enfin, pour permettre à d'autres d'utiliser ces résultats. De plus, si, comme le dit Popper (1965), le propre de toute science est d'être falsifiable, la falsification ne peut s'exercer qu'à partir d'une description substantielle des objets étudiés.

Expliciter, c'est donc décrire. Et décrire, c'est déjà analyser. Lorsque, tout à l'heure, j'ai présenté le fonctionnement de la technique paradigmatique avec l'exemple du premier thème du Prélude I, je procédais déjà à une analyse de ce thème, décrivant, d'une part, les configurations des unités segmentées, et d'autre part, les relations entretenues entre ces unités, et ce, après avoir insisté sur le point de vue dans lequel je me plaçais. La tration était sommaire, mais suffisante je crois, et surtout, indispensable à la compréhension de mon découpage. 


\section{c. Le concept de méta-langage}

Ce concept n'est pas nouveau en musique. La démarche classique l'utilise, entre autres, dans le cadre de l'analyse formelle, pour nommer les grandes sections d'une ouvre et résumer leurs relations d'identité, de similarité ou d'opposition par des formules telles que : $A B$ pour une forme binaire, $A B A$ pour une forme ternaire, etc. Mais la sémiologie en a systématisé l'utilisation à tous les niveaux de l'analyse. Toute unité, qu'elle soit microscopique ou macroscopique, qu'elle soit fondée sur quelque critère que ce soit, se voit dotée d'un symbole abstrait qui la caractérise au sein d'un ensemble. Il est entendu que chaque symbole doit faire référence à une fonction bien spécifiée, de sorte que son utilisation dans des contextes différents ne prête pas à équivoque.

L'emploi d'un méta-langage a donc pour but de désigner de façon économique les caractéristiques des unités dégagées par l'analyse. Favorisant une prise globale des objets soumis à l'observation, il en permet une manipulation commode. Dans le cadre d'une analyse de corpus tout particulièrement, il apparaît de première importance pour la construction d'un modèle combinatoire.

\section{Les étapes décisives de l'enquête}

Dans cette recherche axée sur les thèmes des Préludes du Livre I, et plus spécifiquement, sur ces thèmes tels qu'ils apparaissent lors de leur exposition — c'est-à-dire lors de leur toute première manifestation dans le prélude auquel ils appartiennent -, l'étape originelle a consisté à sélectionner les objets d'étude et à effectuer sur chacun d'eux un découpage paradigmatique basé sur la récurrence mélodico-rythmique, tel qu'on a pu en voir une application tout à l'heure avec l'exemple prélevé dans Danseuses de Delphes. Mais, devant l'abondance de ces thèmes - j'en ai dénombré quarante-six - et l'impossibilité de les traiter tous à la fois, j'ai décidé, dans un premier temps, de restreindre mon champ d'action à ce que j'ai considéré comme étant le premier thème des douzes Préludes. ${ }^{4}$ L'exemple 5 (ci-après) montre quels sont ces thèmes et comment ils ont été analysés selon la technique paradigmatique. ${ }^{5}$ Pour le thème du Prélude I, on se reportera à l'exemple 2 . 
II. Voiles

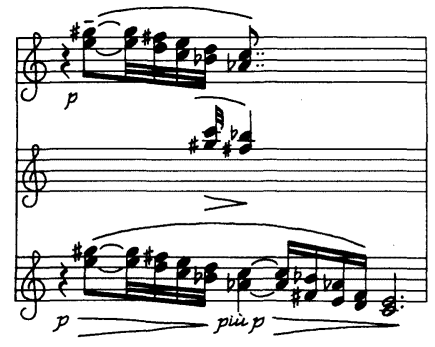

III. Le vent dans la plaine

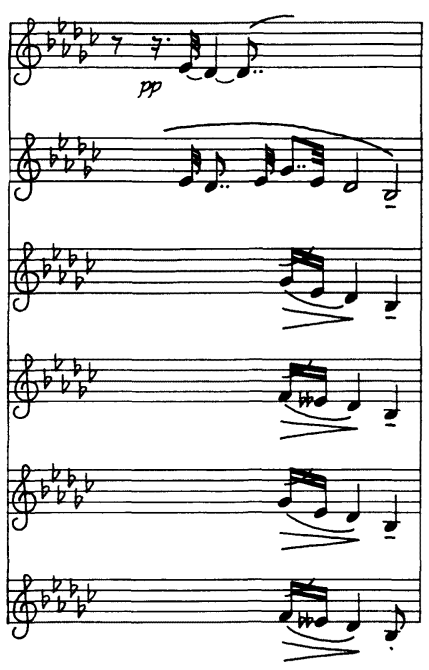

IV. Les sons et les parfums tournent dans l'air du soir

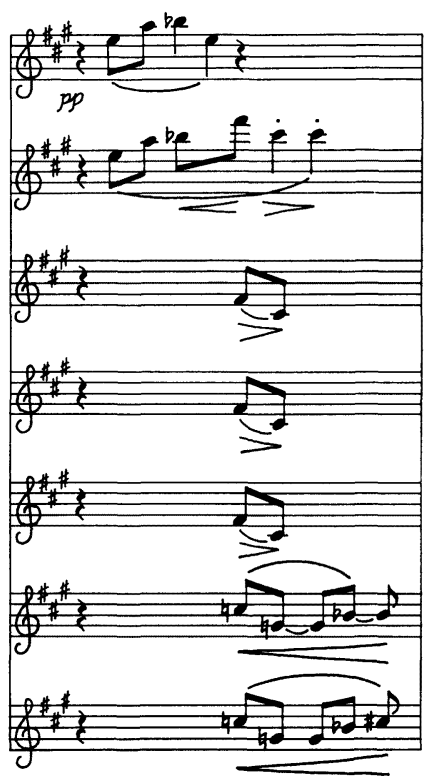



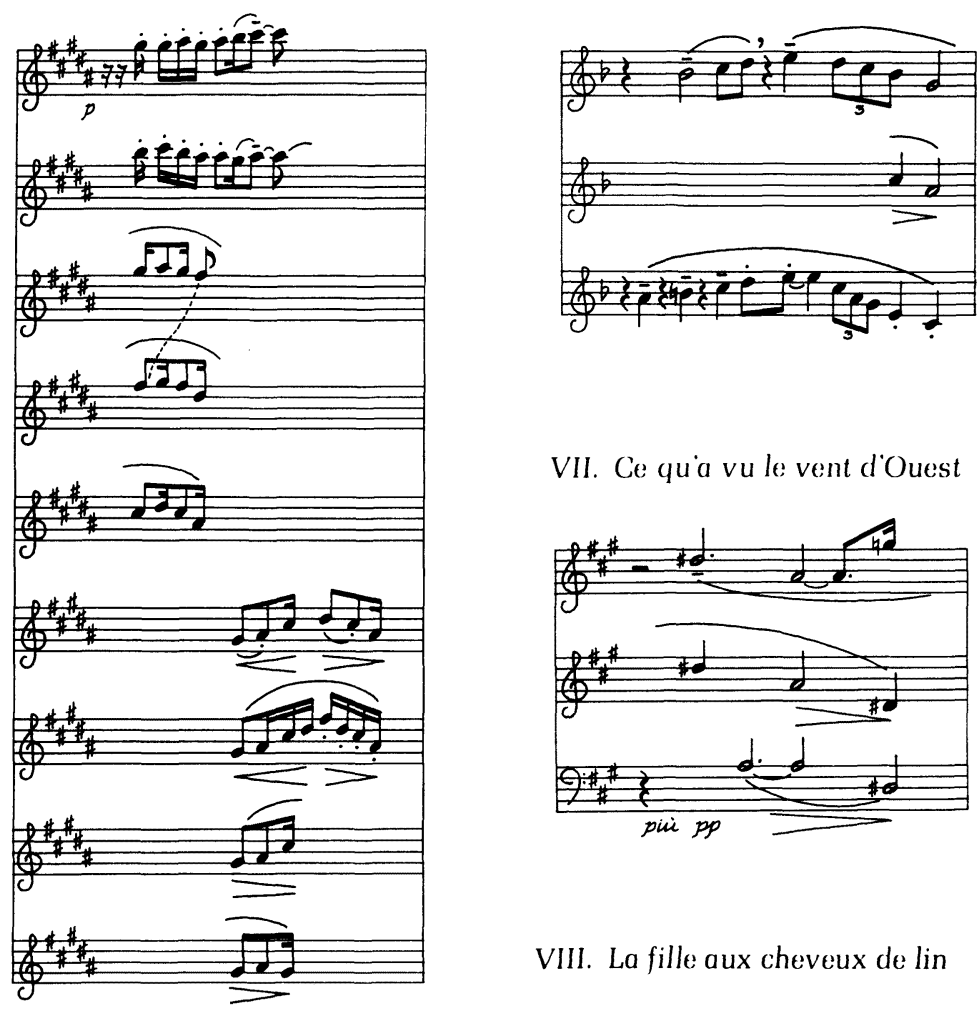

VII. Ce qua vu le vent d'Ouest

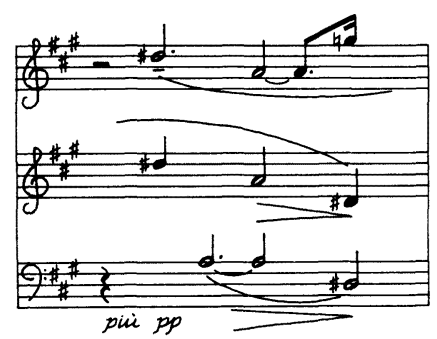

VIII. La fille aux cheveux de lin

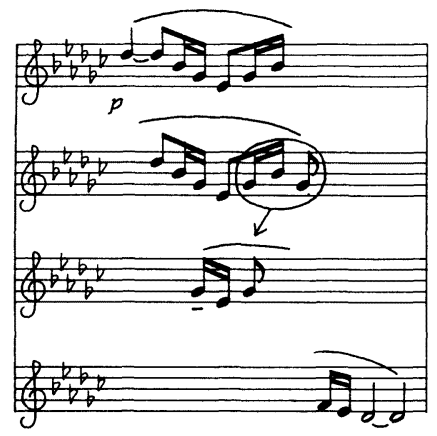


IX. La sérénade interrompue XI. La danse de Puck
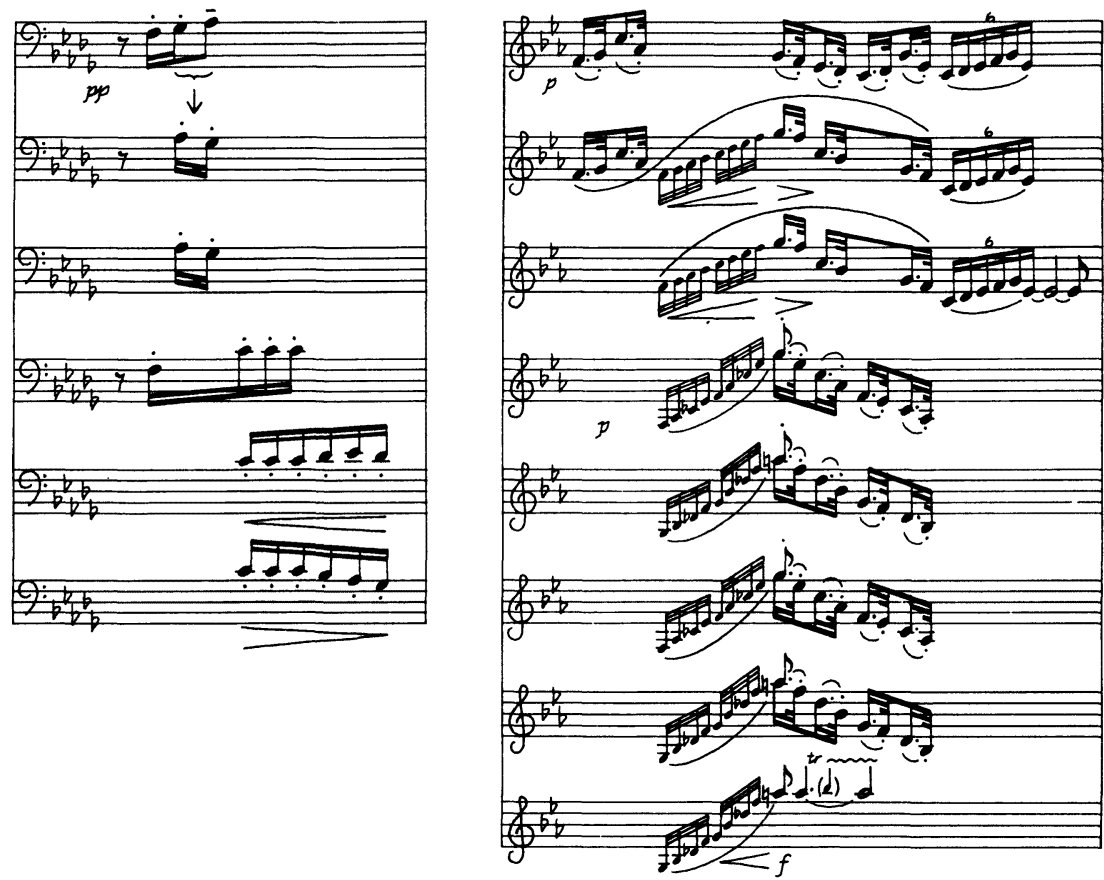

$X$. La cathédrale engloutie

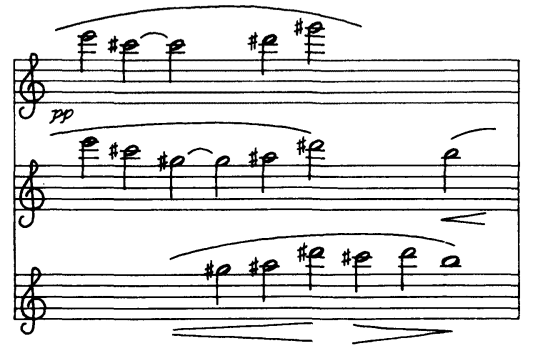

XII. Minstrels

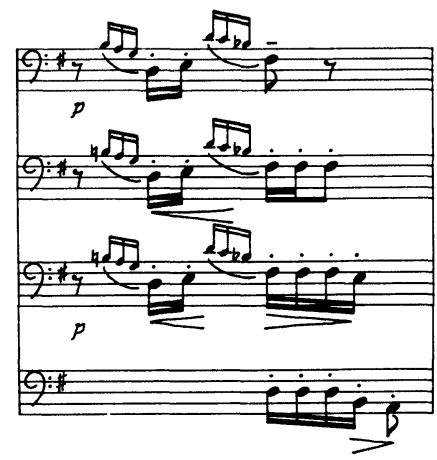


A partir de ce mini-corpus de paradigmes, j'ai envisagé d'élaborer une méthode d'analyse comparative, laquelle, aboutissant à un ensemble de règles, pourrait ensuite être appliquée et vérifiée sur la totalité du corpus thématique. Dans les limites de cet article, c'est essentiellement de l'élaboration de la méthode dont je rendrai compte, en m'appuyant sur les cas les plus courants observés à l'intérieur du mini-corpus cité.

Après avoir longuement examiné les configurations obtenues par la paradigmatisation de chacun de ces douze thèmes, et tenté sur l'ensemble diverses approches comparatives au niveau des intervalles, des échelles, des rythmes, des procédés répétitifs, substitutifs, etc., j'en suis venue à la conclusion que la dimension la plus propice à une confrontation générale était celle de la structure formelle. Il appert en effet, tel que préconisé par la théorie de l'information, que le meilleur moyen de réduire l'information brute est de grouper les signes dans une forme, et d'analyser cette agglomération de signes non tant par rapport à sa nature intrinsèque, que par rapport aux relations structurelles qu'elle entretient avec d'autres unités (voir Moles 1972). C'est dire que, dans l'examen parallèle de mes douze thèmes, les traits mélodiques et rythmiques propres à chacun ont été délaissés au profit d'une caractérisation formelle des unités. Plus spécifiquement, j'ai abordé la question stylistique sous l'angle de la syntaxe, ce mode d'approche impliquant deux notions fondamentales : distribution et fonction.

A partir de ce nouveau point de vue, j'ai repris l'analyse de chacun des paradigmes de mon mini-corpus, en axant cette fois la description, non plus sur le contenu paramétrique des unités découpées, mais sur le rôle joué par chaque unité face à celle qui la précède et celle qui la suit. Reprenons l'exemple du premier thème du Prélude I, celui de l'exemple 3. Le genre d'observation fait sur ce thème - comme sur chacun des onze autres - repose sur la question suivante : dans la comparaison de deux unités successives, comment se comporte la seconde unité vis-à-vis de la première? On se reportera à la première colonne de l'exemple 6 , inséré plus loin.

Considérons les deux unités initiales, respectivement symbolisées par $x$ et $x^{\prime}$, symboles dont je reparlerai plus loin. $x^{\prime}$ reprend $x$ avec une légère modification à la voix inférieure. Au niveau formel, il s'agit donc d'une répétition de $x$. 
Voyons ensuite les unités $x^{\prime}$ et $u$. $u$ ne retient de $x^{\prime}$ que les notes finales de la voix supérieure auxquelles elle greffe une rythmique empruntée à la voix inférieure. C'est une réduction de $x^{\prime}$.

Puis u est repris deux fois, sans transformation aucune. Il y a donc triplication de l'unité de réduction.

Enfin, nous aboutissons à l'unité terminale $(u+)$ du thème : celle-ci transforme u par substitution (sol-fa au lieu de sol-

la) et par adjonction (l'échappée si bémol).

En résumant l'étude effectuée sur ce thème, on obtient l'itinéraire formel suivant:

1. Une donnée de départ

2. Une répétition intégrale de la donnée première avec ajout

3. Rétention de la seule finale de 2 .

4. 5. Reprise exacte de 3

6. Un substitut de 3,4 et 5 .

Ce type d'analyse fonctionnelle entre unités successives a été appliqué à chacun des onze autres thèmes. Au cours de ce travail - qu'il m'est impossible de rapporter en détail ici - j'ai constaté avec étonnement que les procédés formels utilisés étaient similaires d'un thème à un autre, et, qui plus est, qu'ils semblaient intervenir dans un ordre quasi-systématique. J'ai donc procédé à la mise en série des analyses individuelles de mes douze thèmes. Cherchant à illustrer de façon synthétique, mais en même temps complète, la constance au niveau des procédés structurels, j'ai tenté d'imaginer un tableau général à deux dimensions qui puisse témoigner à la fois de la facture de chaque thème et de la facture comparée des thèmes entre eux. C'est ainsi que j'ai conçu le tableau de l'exemple 6 (ci-après). Voyons-en le fonctionnement.

Ce tableau se compose de douze colonnes, chacune rendant compte de l'organisation fonctionnelle et distributionnelle des unités d'un même thème. On peut ainsi, en parcourant chaque colonne de haut en bas, faire une lecture isolée de chaque thème. C'est l'axe d'équivalence vertical dont je parlais tout à l'heure en expliquant la technique paradigmatique. Mais on peut aussi, en passant d'une colonne à la suivante, effectuer une lecture horizontale et comparée des thèmes - il s'agit maintenant d'un axe d'équivalence couché, construit selon le principe suivant : les unités apparaissant sur une même ligne sont reconnues comme équivalentes quant à la fonction qu'elles assument dans 


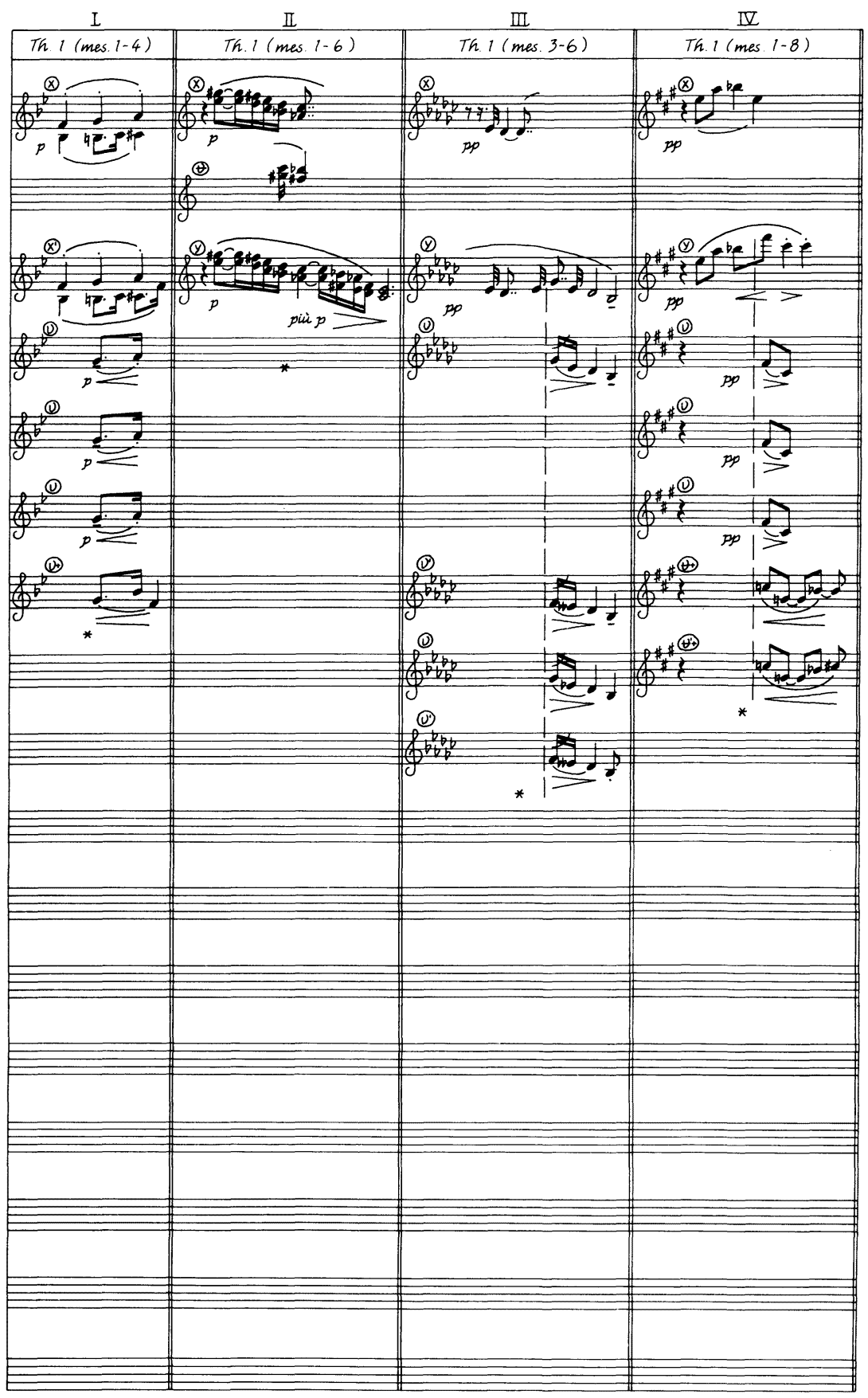

Exemple 6(i) 
I

II

VII

VIII

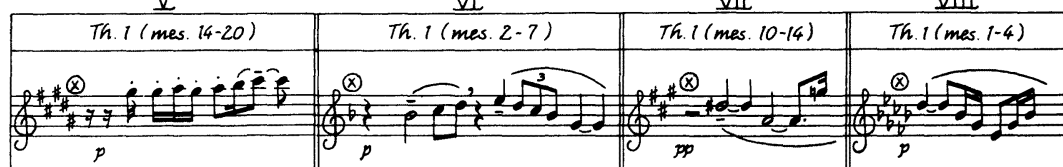

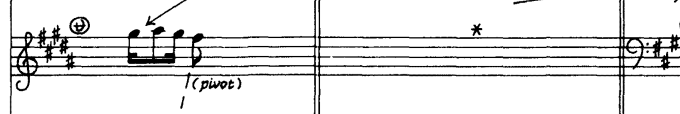
piu p

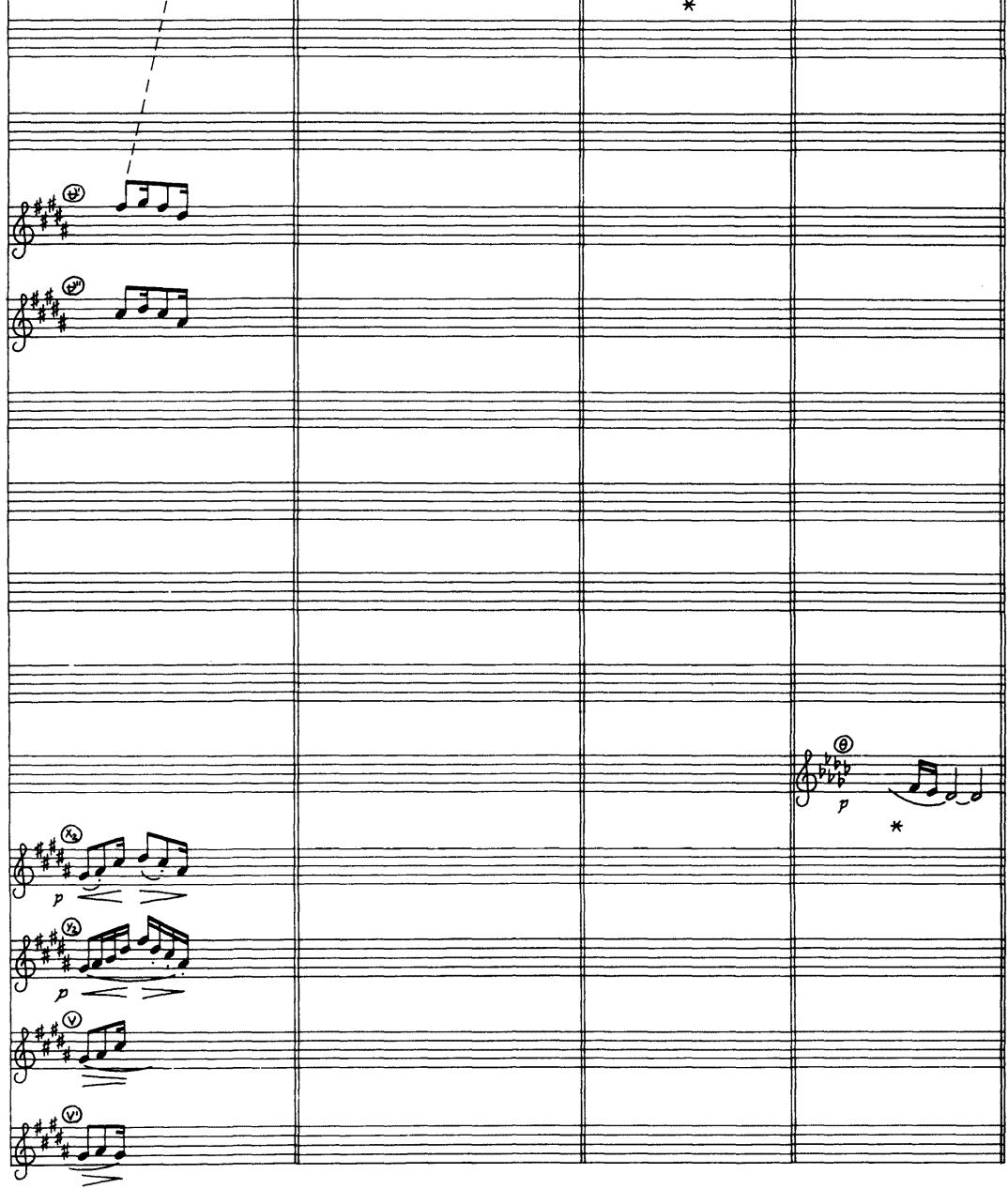

Exemple 6(ii) 


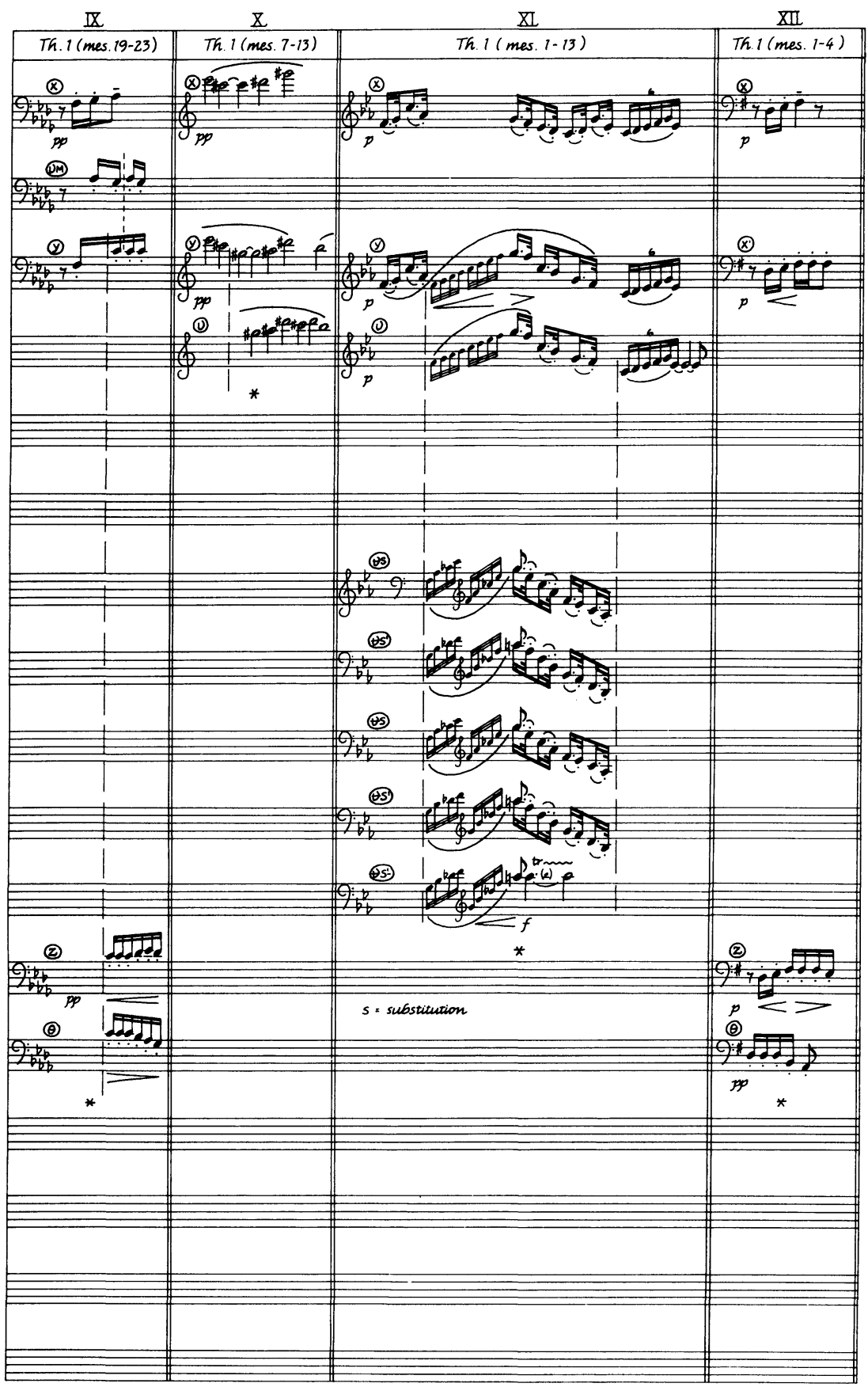

Exemple 6(iii) 
leur thème respectif. Ce tableau constitue donc un paradigme (horizontal) des paradigmes (verticaux).

Abordons maintenant la question du méta-langage, laquelle est d'une importance capitale dans cette entreprise stylistique, puisque chaque symbole utilisé doit, d'un thème à l'autre - c'est donc dire dans des contextes mélodicorythmiques passablement différents - toujours faire référence à une même définition. De plus, étant donné que c'est à partir de ces symboles que seront élaborées des règles ainsi qu'un modèle pour la génération formelle d'un thème debussien, il importe que le méta-langage soit établi dans la plus grande rigueur. En effet, une analyse finale se fondera essentiellement sur ce métalangage, lequel, comme je le disais précédemment, constitue le moyen économique par excellence pour opérer un travail synthétique.

En considérant les trois pages du tableau de l'exemple 6, voyons les unités-types que la mise en série m'a permis de dégager à travers les douze thèmes. J'entends par unité-type, une unité dotée d'une fonction spécifique, et par conséquent d'un symbole spécifique, qui apparaît dans la majorité des thèmes. J'ai relevé trois unités-types : $x, x^{\prime}$ ou $y, u$ ou $\mathrm{u}$. La définition que je donnerai de chacune de ces unités est fondée sur des traits syntaxiques, lesquels, s'appuyant sur la notion de relations formelles entre unités, se révèlent en même temps être des règles d'élaboration thématique.

\section{Première unité du thème : $x$}

Cette unité est délimitée d'après un critère de répétition : elle est toujours reprise à partir de son début par l'unité qui lui succède immédiatement - sauf exception, et nous y reviendrons - , soit dans sa totalité, soit en partie. La fonction de $\mathrm{x}$ consiste donc à agir comme tête de thème, non seulement parce qu'elle figure en tête de paradigme, mais parce que, en tant qu'organisation de hauteurs et de valeurs, elle constitue l'amorce d'un geste, d'une idée qui se verra progressivement développée par les unités suivantes, selon un processus hautement répétitif axé sur les quatre possibilités que voici : répétition identique / répétition avec extension (ou ajout) / répétition avec suppression / répétition avec substitution. 
Deuxième unité du thème : symbolisée tantôt par $x$, tantôt par $y$, pour des raisons que j'explicite plus bas.

Il s'agit d'une unité répétitive de $x$, succédant, de manière générale, immédiatement à celle-ci, mais passible d'un léger délai pour se manifester plutôt comme troisième unité - ce dernier cas intervenant dans le thème des Préludes II, VI et IX. Sa fonction consiste à reprendre l'énoncé de la tête de thème, mais en lui apportant toujours une modification qui, la plupart du temps, a lieu en finale. $x^{\prime}$ ou y débute donc sur le modèle de $x$ - sauf exception, tel que cela apparaît dans le thème du Prélude V. Quand l'unité répétitive de x porte le symbole $x^{\prime}-$ voir dans les Préludes I, VIII et XII —, c'est qu'elle refait intégralement le trajet de la tête de thème, pour la modifier par un élément qui n'en altérera jamais l'ambitus. Quand l'unité répétitive de $\mathrm{x}$ s'appelle $\mathrm{y}$ - voir dans les Préludes II, III, IV, VI, VII, IX, X, XI -, c'est que, reprenant la tête de thème soit intégralement, soit partiellement - dans ce dernier cas, l'initiale de $\mathrm{x}$ est toujours conservée -, elle effectue une modification sur le plan de l'ambitus : celui-ci est élargi, soit par ajout, soit par substitution de notes.

Il est à noter que ces deux types d'unités $\mathrm{x}^{\prime}$ ou y se retrouvent dans tous les thèmes. Dans le Prélude V, la seconde unité se nomme exceptionnellement $x^{\prime} m$, l'indice $m$ faisant référence à la quasi-rétrogradation mélodique effectuée par rapport à $\mathrm{x}$.

\section{Troisième unité du thème : nommé u ou u}

Il s'agit d'une unité de réduction, présente dans tous les thèmes sauf dans celui du Prélude XII. Son rôle consiste à ne redonner que le fragment terminal de l'unité qui la précède immédiatement. Dans la majorité des cas, ce type d'unité intervient sitôt après $\mathrm{x}^{\prime}$ ou $\mathrm{y}$, en tant que troisième unité - voir dans les Préludes I, III, IV, VII, VIII, X, et XI -; il reprend les hauteurs finales de l'unité qui précède, ces hauteurs coïncidant, dans les cas où la deuxième unité s'appelle y - voir dans les Préludes III, IV, VII, X et XI - à l'ajout effectué par cette deuxième unité en regard de la tête de thème. ${ }^{7}$

Généralement, l'unité de réduction porte le symbole u; elle apporte toujours, en même temps que la réduction, une quelconque transformation rythmique, à laquelle peut s'ajouter une modification sur le plan du registre - comme dans les 
Préludes IV et VII. Parfois, l'indice m lui est accolé (Prélude IX) : c'est le signe d'une rétrogradation mélodique - ici, dupliquée. Quand l'unité de réduction porte le symbole $\mathbf{t}$ (Préludes II, V, VI,), c'est que le fragment final de l'unité qui précède a été transposé par rapport aux hauteurs originales le transfert aux octaves supérieures ou inférieures n'étant pas considéré comme une véritable transposition.

Venons-en maintenant à la distribution de cette unité. Tel que mentionné plus haut, u ou ses variantes um/u) se manifeste normalement comme troisième unité. Dans trois Préludes cependant (II, VI et IX), elle ne succède pas à $\mathrm{x}^{\prime}$ ou $\mathrm{y}$, mais bien à $x$, la tête de thème, et pratique donc la réduction de celle-ci.

A l'aide des trois unités-types $x, x^{\prime}$ ou $y$, et $u$ ou ses variantes, dégagées dans onze Préludes sur douze, il est possible de proposer un canevas de base à la structure du premier thème, puisque les thèmes des Préludes II, VI, VII et X répondent à cette architecture. Pour avoir une saisie plus globale de la question, nous nous servirons maintenant du tableau suivant (ex. 7), qui, tout en respectant les deux dimensions de lectures verticale et horizontale du tableau de l'exemple 6, ne conserve plus que les symboles du méta-langage utilisé.

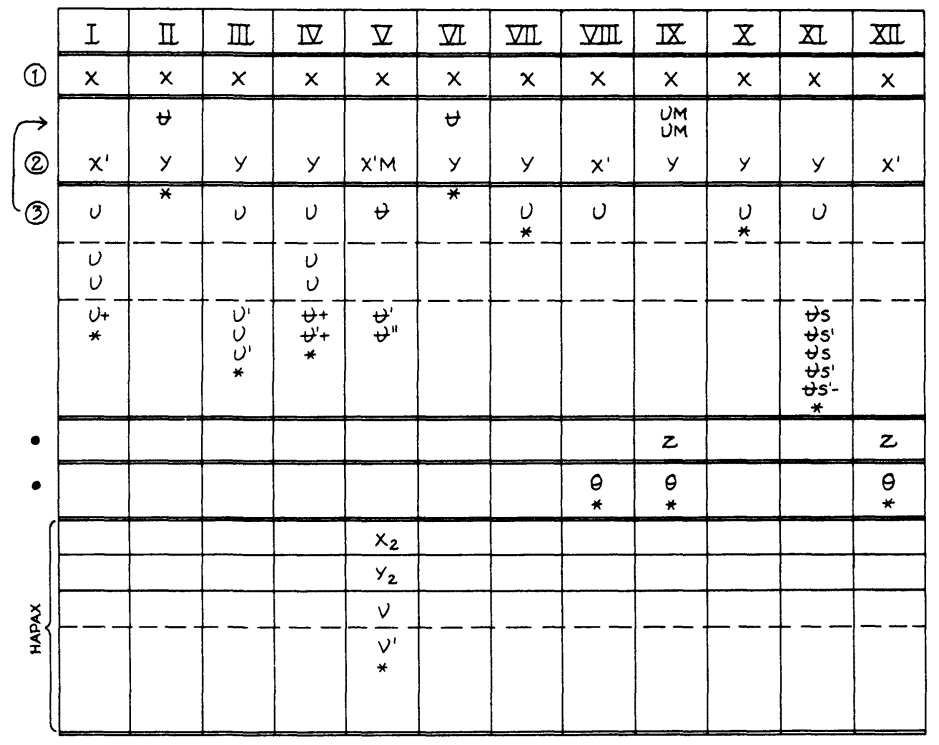


Ayant examiné le type et la distribution des trois premières unités, voyons le modèle de base auquel nous aboutissons, lequel est susceptible de se réaliser sous deux modes différents. ${ }^{8}$

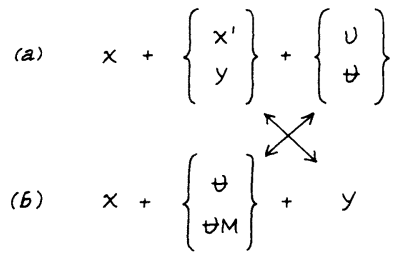

Exemple 8

En a), nous avons ce que, pour des raisons quantitatives, j'ai. appelé le modèle fondamental; on retrouve en effet le schéma $x$ suivi de $\mathrm{x}^{\prime}$ ou $\mathrm{y}$, à son tour suivi de $\mathrm{u}$ ou $\mathrm{u}$, dans 7 thèmes sur 12 , à savoir celui des Préludes I, III, IV, VII, VIII, X et XI.

En b), nous avons le modèle optionnel, tel qu'il apparaît dans le thème des Préludes II, VI et IX. Il s'agit d'une variante distributionnelle du modèle fondamental, variante selon laquelle l'ordre des deuxième et troisième unités est interverti : $x$ immédiatement suivi de $\mathrm{u}$ ou um, à son tour suivi de $\mathrm{y}$.

On pourrait bien sûr effectuer des remarques plus fines quant aux particularités de ces deux modèles de base. A savoir qu'ils ne se distinguent pas uniquement sur le plan de la distribution des unités 2 et 3 , mais également sur celui de la facture formelle des dites unités. Par exemple, à l'intérieur du modèle fondamental, l'unité de réduction, qui occupe la troisième place, est passible de revêtir soit le symbole $u$, soit le symbole $\mathrm{u}$, tandis qu'à l'intérieur du modèle optionnel, cette même unité, intervenant en second, n'est jamais dotée du symbole $u$, mais plutôt de u ou de um. C'est dire que, dans ce second cas, et tel que défini précédemment dans l'emploi de mon méta-langage, la fonction de réduction est toujours liée à une transformation mélodique du type transposition ou rétrogradation, transformation qui n'est pas du tout obligatoire dans le premier cas.

Ce genre de remarques par lesquelles on peut raffiner l'analyse a certes occupé une large part dans ma recherche, mais je n'y insisterai pas davantage dans cet exposé, mon objectif consistant ici à présenter dans ses grandes lignes le modèle formel minimal des thèmes de mon mini-corpus. Pourquoi ai-je considéré ce modèle comme minimal? Parce que, dans un 
premier sens, prenant en charge la totalité de certains thèmes (cf. II, VI, VII et XI), il suffit à en illustrer la structure; il est minimal aussi en ce qu'il indique quels sont les traits syntaxiques qui caractérisent généralement le début d'un thème.

Au-delà du schéma de base, le thème peut se prolonger selon différentes modalités. Je parlerai de la formule la plus courante, celle qu'on trouve dans le thème des Préludes I, III, IV, $\mathrm{V}$ et XI (cf. le tableau de l'exemple 6) : à la suite du modèle fondamental $-\mathrm{x} / \mathrm{x}^{\prime}$ ou $\mathrm{y} / \mathrm{u}$ ou $\mathrm{u}-$, la troisième unité, dite unité de réduction, subit un traitement répétitif. La répétition peut avoir cours jusqu'à cinq fois, de manière identique ou non. Dans le cas de transformations, celles-ci reposent principalement sur la transposition et sur l'alternance entre état original et état transformé. Le principe majeur d'extension du thème, au-delà de ses trois premières unités, consiste donc à exploiter l'unité de réduction.

Les caractéristiques formelles dégagées à travers les douze thèmes observés - et dont j'ai exposé ici les plus récurrentes ont donné lieu, outre la construction de modèles combinatoires, à la formation de dix règles génératives axées sur la fonction et la distribution d'unités.

\section{Un aperçu des prolongements de la recherche}

C'est à l'aide de la méthode élaborée dans un cadre aussi restreint que ce mini-corpus constitué par le premier thème des douze préludes qu'il m'a été loisible de poursuivre aisément la recherche. Ayant accumulé suffisamment de renseignements sur un certain faire thématique, j'étais maintenant en mesure d'ouvrir mon champ analytique, pour vérifier la pertinence ou la non-pertinence des résultats obtenus sur l'ensemble du corpus thématique. Car, une question me préoccupait: le squelette structurel du premier thème est-il exclusif à celui-ci, ou sert-il d'assise à d'autres thèmes? S'il est typique du seul premier thème, cela démontrerait qu'il $\mathrm{y}$ a permanence dans certain processus de commencement des préludes en ce qui concerne la structuration formelle de la mélodie. Par contre, si ce squelette déborde le contexte du premier thème, sa pertinence est d'ordre plus général, et il y a lieu d'oublier l'hypothèse première.

J'ai donc procédé, un à un, à l'étude formelle de tous les autres thèmes du Livre I, considérés lors de leur exposition. Nombre d'entre eux sont venus grossir les rangs du modèle 
présenté plus haut, indiquant que ce modèle n'est pas exclusif au premier thème, mais qu'il est statistiquement prépondérant à travers le corpus. ${ }^{9}$ Que les données de ce modèle aient été réalisées consciemment ou non par Debussy, il n'en demeure pas moins qu'elles nous révèlent, comme facette de son style, la présence de structures formelles relativement fixes sous des lignes mélodiques variées quant aux échelles, hauteurs, intervalles, contours, etc. Face cachée uniforme versus face visible multiple : la dialectique du "fugitif » et du "durable », du " pareil » et du " différent ", évoquée au début de cet article, ne se joue certainement pas que là ; mais elle me semble y avoir une part non négligeable. ${ }^{10}$ 


\section{NOTES}

1. Un exemple facile et concret: le cas de la gamme par tons chez Debussy. Nombre de mélodies du compositeur, pourtant fort différentes sur le plan des intervalles, de la courbe, de l'ambitus, etc., ont en commun d'être fondées sur ce principe de division de l'octave en secondes majeures.

2. Il s'agit d'un condensé de la définition fournie par Blom (1954).

3. G.G. Granger discute de la question du concept semi-intuitif dans Pensée formelle et sciences de l'homme (1967).

4. Décision arbitraire, certes, mais reposant sur un type d'argument excellement bien formulé par Umberto Eco : « Ne peut-on pas dire pour ces œuvres d'art, comme le fait le savant pour la situation expérimentale, que la connaissance incomplète d'un système est une composante essentielle de sa formulation " $(1965: 30)$ ?

5. Ceux des Préludes IV, VII et X sont, dans le texte, des thèmes polyphoniques. Ils ont été réduits ici à leur ligne principale pour rendre la recherche plus aisée - la réduction n'affectant aucunement, comme on le verra par la suite, le phénomène sur lequel je me suis penchée.

6. Pour le thème du Prélude I, se reporter à l'ex. 3.

7. Dans mon tableau, la ligne pointillée verticale marque cet ajout.

8. Dans chacune des figures présentées, les accolades indiquent qu'il y a possibilité de choix entre deux types d'unités.

9. Sur les quarante-six thèmes dénombrés, vingt-huit répondent en effet à ce modèle. Quant aux dix-huit thèmes restants, ils ont donné lieu à la construction. de cinq autres schémas structurels, respectivement fondés sur une distribution spécifique d'unités-types parmi lesquelles figurent ou ne figurent pas, selon les cas, celles qui régissent la configuration du modèle prépondérant.

10. Le présent article s'inscrit dans le cadre de recherches sémiologiques sur Debussy, subventionnée par le Conseil des Arts puis par le Conseil de Recherches en Sciences Humaines du Canada et l'Université de Montréal entre 1974 et 1980. 


\section{RÉFÉRENCES}

BARTHES, R., 1963 : Sur Racine. Paris : Seuil.

BLOM, E.,

1954 : "Theme ", in Grove's Dictionary of Music and Musicians. 5ième éd., Eric Blom, éd. London: Macmillan, VIII, 409-10.

ECO, U.,

1965 : L'œuvre ouverte. Paris : Seuil.

GRANGER, G.G.,

1967 : Pensée formelle et sciences de l'homme. Paris : Aubier. MESSAGER, J.A.,

1938 : L'enfance de Pelléas : Lettres de Claude Debussy à André Messager, recueillies et annotées par Jean-André Messager. Paris : Dorbon.

MOLES, A.,

1972 : Théorie de l'information et perception esthétique. Paris : Denoël.

POPPER, K.,

1965 : The Logic of Scientific Discovery. New York: Harper Torchbooks. 